Bio - grafía. Escritos sobre la Biología y su Enseñanza. ISSN 2027-1034

Edición Extraordinaria. p.p. 237 - 244

Memorias del IX Encuentro Nacional de Experiencias en Enseñanza de la Biología y la

Educación Ambiental. IV Congreso Nacional de Investigación en Enseñanza de la

Biología.

\title{
CLUB AMBIENTAL COMO AMBIENTE DE APRENDIZAJE PARA RECONOCIMIENTO Y TRANSFORMACIÓN DE REALID ADES AMBIENTALES: Un caso práctico desde el Colegio Cundinamarca I.E.D.
}

\section{Julio Cesar Donato $\mathbf{M}^{1}$}

\section{RESUMEN}

El presente documento describe el desarrollo e implementación de un ambiente de aprendizaje denominado club ambiental en el colegio Cundinamarca de la localidad de Ciudad Bolívar en Bogotá, desde sus inicios en 2009 hasta el día de hoy; señalando los procesos metodológicos llevados a cabo así como las diferentes actividades y logros conseguidos en estos 8 años. Se destaca igualmente el impacto que este club ha tenido en los procesos curriculares y extracurriculares al interior de la institución en términos del reconocimiento del entorno ambiental particular del Colegio Cundinamarca así como las alternativas de solución para el control y/o mitigación de dichas problemáticas ambientales.

\section{PALABRAS CLAVE}

Club ambiental, Investigación, Problemática, Intervención, Momentos.

\section{ABSTRACT}

This document describes the development and implementation of a learning environment called the environmental club in the Cundinamarca school in the town of Ciudad Bolívar in Bogotá, from its beginnings in 2009 to today; pointing out the methodological processes carried out as well as the different activities and achievements achieved in these 8 years. It also highlights the impact that this club has had on the curricular and extracurricular processes within the institution in terms of the recognition of Cundinamarca school particular environment as well as alternative solutions for the control and / or mitigation of these environmental issues.

\section{KEY WORD}

Environmental Club, Research, Problems, Intervention, Moments.

\footnotetext{
1 Julio Cesar Donato M. Docente Colegio Cundinamarca. Licenciado en Biología UPN, Especialista en Pedagogía de la Recreación Ecológica Fundación Los Libertadores, Candidato a Magister en Administración y planificación educativa UMECIT Panamá.
} 
Bio - grafía. Escritos sobre la Biología y su Enseñanza. ISSN 2027-1034

Edición Extraordinaria. p.p. 237 - 244

Memorias del IX Encuentro Nacional de Experiencias en Enseñanza de la Biología y la Educación Ambiental. IV Congreso Nacional de Investigación en Enseñanza de la Biología.

\section{INTRODUCCIÓN}

EI ECOCLUB BLUE PLANET del Colegio Cundinamarca ubicado en la Localidad de Ciudad Bolívar en Bogotá, surge el 2008 como iniciativa, producto de la ubicación ambientalmente crítica del colegio, el cual está rodeado de fábricas y del interés de jóvenes de los ciclos $3\left(5,6^{\circ}\right.$ y 7$)$ con respecto a su realidad ambiental; al año siguiente (2009) se vincula la ONG oPepa y cartón de Colombia con capacitaciones relacionadas con la organización de un ecoclub; desde entonces se han realizado actividades de apoyo y fortalecimiento al PRAE del colegio y acciones de sensibilización ambiental en celebraciones como el día de la tierra, del agua y del ambiente. Durante el periodo comprendido entre 2008 a 2014 los encuentros con los jóvenes de los grados $5^{\circ}$ a $9^{\circ}$ se han realizado los días sábados de 9:00 a $12 \mathrm{~m}$. estos incluyen actividades de capacitación, lúdicas y audiovisuales principalmente; de igual manera han participado en diferentes eventos locales, distritales y nacionales destacándose en los foros institucionales y locales en los años 2011 y 2012.

A partir del año 2015 y con el programa 40x40 se genera una nueva forma de concebir el club ambiental, esto es como un centro de interés, el cual proviene,...del enfoque pedagógico activo, basado en la observación de los niños y en su aprendizaje. Se desarrollan en el contexto de la "escuela por la vida y para la vida" de Decroly que permite a los maestros y educandos abordar un conjunto de conocimientos culturales básicos, en torno a un tema central, elegido en función de las necesidades e intereses de los estudiantes (SED 2014). Este club ambiental se implementa para desarrollar en contrajornada los días martes y jueves de 9:00 a.m. a 11:30 a.m; como un AMBIENTE DE APRENDIZAJE es decir..."un proceso pedagógico que se adapta a las necesidades y los contextos particulares y sociales de las y los estudiantes. En él se utilizan recursos didácticos para crear condiciones y espacios interactivos, creativos, intencionados y lúdicos, en los que los participantes desempeñan roles de acuerdo a las circunstancias y se generan sistemas de conocimiento que facilitan su vivencia práctica" (SED, 2015). Actualmente el grupo del club ambiental está conformado por 20 estudiantes que cursan los grados de $5^{\circ}$ a $7^{\circ}$ (Ciclo 3 ), Estos estudiantes se caracterizan por su curiosidad, proactividad, deseos de aprender y trabajar por el ambiente.

\section{METODOLOGÍA}

La metodología de trabajo implementada en este ambiente de aprendizaje denominado Club Ambiental es del tipo acción- participación, bajo el modelo de pedagogía critica, el cual está orientado en otra forma de educación que incite a los estudiantes a cuestionar, a no asumir verdades absolutas sin antes reflexionar y complementar los conocimientos recibidos en las aulas, y de esta manera se permita un pensamiento crítico para la construcción de nuevas realidades y trabajar por la transformación de realidades y la construcción de nuevas oportunidades de vida y de conocimiento....en pro de una mejor calidad de vida; relacionando con lo que dice Freire en busca de la liberación de ciertas modelos sociales que lo oprimen; su objetivo es que el estudiante reflexione y se interese en la búsqueda de solución a los diferentes problemas en los que está inmerso en su casa, barrio, colegio y comunidad circundante; y de esta manera reflexiva y progresivamente toma decisiones que le permite dar solución a diferentes conflictos con 
Memorias del IX Encuentro Nacional de Experiencias en Enseñanza de la Biología y la Educación Ambiental. IV Congreso Nacional de Investigación en Enseñanza de la Biología.

el diseño de un diagnóstico de problemas ambientales institucionales, a partir del cual se dialoga y generan propuestas de intervención orientadas a la mitigación y/o control de dichas problemáticas. La metodología se desarrolla en 4 momentos específicos:

\begin{tabular}{|c|c|c|}
\hline MOMENTO & OBJETIVO & ACTIVID ADES Y RESULTADOS ESPERADOS \\
\hline $\begin{array}{l}\text { 1. Pensarse y } \\
\text { pensarnos }\end{array}$ & $\begin{array}{l}\text { Reconocer y reflexionar } \\
\text { sobre los escenarios } \\
\text { ambientales presentes en } \\
\text { el colegio Cundinamarca } \\
\text { en términos de } \\
\text { potencialidades r y } \\
\text { dificultades. }\end{array}$ & $\begin{array}{l}\text { Identificar y reconocer el contexto ambiental } \\
\text { intra y extra institucional. } \\
\text { Determinar las causas y consecuencias de los } \\
\text { principales problemas ambientales del colegio }\end{array}$ \\
\hline $\begin{array}{l}\text { 2. Diálogo de } \\
\text { saberes }\end{array}$ & $\begin{array}{l}\text { Desarrollar habilidades y } \\
\text { actitudes de compromiso } \\
\text { hacia el entorno ambiental } \\
\text { del colegio, que les } \\
\text { permitan a los } \\
\text { estudiantes, proponer y } \\
\text { diseñar estrategias de } \\
\text { solución a los problemas } \\
\text { ambientales }\end{array}$ & $\begin{array}{l}\text { Generar posturas críticas y propositivas frente a } \\
\text { las problemáticas ambientales detectadas } \\
\text { Desarrollar procesos de sensibilización } \\
\text { mediante talleres y capacitaciones sobre el uso } \\
\text { racional y sostenible de los recursos naturales. } \\
\text { Reconocer formas de manejo ambiental } \\
\text { sostenible mediante salidas pedagógicas a } \\
\text { escenarios naturales }\end{array}$ \\
\hline $\begin{array}{l}\text { 3. Transformando } \\
\text { Realidades }\end{array}$ & $\begin{array}{l}\text { Realizar actividades de } \\
\text { impacto en la comunidad } \\
\text { educativa tendientes a } \\
\text { fomentar la sensibilización } \\
\text { y cambio de hábitos y } \\
\text { actitudes ambientales a } \\
\text { favor del entorno. }\end{array}$ & $\begin{array}{c}\text { Diseñar e implementar un aula virtual } \\
\text { relacionada con los conocimientos adquiridos y } \\
\text { aplicados y las actitudes adoptadas por los } \\
\text { estudiantes durante la realización del } \\
\text { diagnóstico ambiental } \\
\text { Fomentar la sensibilización ambiental frente al } \\
\text { uso racional de los recursos: agua, energía y } \\
\text { suelo, y la disposición adecuada de residuos } \\
\text { sólidos en la institución. } \\
\text { Fortalecer el proyecto ambiental escolar } \\
\text { mediante la participación activa de jóvenes } \\
\text { líderes ambientales en las actividades } \\
\text { planteadas }\end{array}$ \\
\hline $\begin{array}{l}\text { 4. Reconstruyendo } \\
\text { saberes }\end{array}$ & $\begin{array}{l}\text { Divulgar a la comunidad } \\
\text { educativa la información } \\
\text { recolectada durante el } \\
\text { diagnóstico así como el } \\
\text { desarrollo, ejecución y } \\
\text { resultados del proceso de } \\
\text { implementación de } \\
\text { acciones de solución a los } \\
\text { problemas ambientales }\end{array}$ & $\begin{array}{l}\text { Socializar los resultados significativos obtenidos } \\
\text { durante la experiencia mediante diferentes } \\
\text { estrategias } \\
\text { Sistematizar la información obtenida en un } \\
\text { pendón, en el cual se identifique las situaciones } \\
\text { problema y acciones de solución realizadas }\end{array}$ \\
\hline
\end{tabular}


Bio - grafía. Escritos sobre la Biología y su Enseñanza. ISSN 2027-1034

Edición Extraordinaria. p.p. 237 - 244

Memorias del IX Encuentro Nacional de Experiencias en Enseñanza de la Biología y la Educación Ambiental. IV Congreso Nacional de Investigación en Enseñanza de la Biología.

\begin{tabular}{|l|l|l|}
\hline & identificados. & \\
\hline
\end{tabular}

momentos específicos, anteriormente descritos:

\begin{tabular}{|c|c|c|c|}
\hline \multicolumn{4}{|c|}{ Momento 1: Pensarse y pensarnos } \\
\hline $\begin{array}{l}\text { Ses } \\
\text { ión }\end{array}$ & Nombre & Objetivo & Actividad \\
\hline 1 & Presentación & $\begin{array}{l}\text { Socializar la } \\
\text { propuesta } \\
\text { pedagógica y } \\
\text { metodológica del } \\
\text { centro de interés }\end{array}$ & $\begin{array}{l}\text { Presentación del centro de interés e } \\
\text { identificación de intereses y expectativas de los } \\
\text { estudiantes mediante dinámica rompehielos. }\end{array}$ \\
\hline 2 & $\begin{array}{c}\text { MAPA AMBIENTAL } \\
\text { COLEGIO }\end{array}$ & \multirow{3}{*}{$\begin{array}{l}\text { Identificar } r \text { el } \\
\text { contexto ambiental } \\
\text { intra y extra } \\
\text { institucional }\end{array}$} & $\begin{array}{l}\text { Elaboración estudiantil grupal del mapa del } \\
\text { colegio, localizando en éste los lugares de } \\
\text { relevancia ambiental. Juego de mesa Colegio } \\
\text { Cundinamarca }\end{array}$ \\
\hline 3 & $\begin{array}{l}\text { RECORRIDO } \\
\text { EXTERNO } \\
\text { COLEGIO }\end{array}$ & & $\begin{array}{l}\text { Recorrido a los alrededores del colegio con el } \\
\text { fin de realizar un mapa de riesgos ambientales } \\
\text { externos. }\end{array}$ \\
\hline 4 & $\begin{array}{c}\text { VISITAS A } \\
\text { EMPRESAS }\end{array}$ & & $\begin{array}{l}\text { Visitas a algunas fábricas cercanas para } \\
\text { identificar las actividades del proceso productivo } \\
\text { que generen impacto y proponer medidas de } \\
\text { mitigación y/o control ambiental: Colcueros, } \\
\text { Filmtex y Minipack. }\end{array}$ \\
\hline 5 & $\begin{array}{l}\text { ENCUESTAS Y } \\
\text { ENTREVISTAS } \\
\text { AMBIENTALES }\end{array}$ & \multirow{3}{*}{$\begin{array}{lr}\text { Determinar las } & \text { las } \\
\text { causas } & \text { y } \\
\text { consecuencias de } \\
\text { los principales } \\
\text { problemas } \\
\text { ambientales del } \\
\text { colegio }\end{array}$} & $\begin{array}{l}\text { Diseño, construcción y aplicación de encuestas } \\
\text { enfocadas a la detección de problemas } \\
\text { ambientales del colegio }\end{array}$ \\
\hline 6 & $\begin{array}{l}\text { APLICACIÓN DE } \\
\text { ENTREVISTAS Y } \\
\text { ENCUESTAS }\end{array}$ & & $\begin{array}{l}\text { Entrevistas a miembros de la comunidad } \\
\text { educativa con el propósito de indagar sobre las } \\
\text { problemáticas ambientales del colegio } \\
\text { identificadas por ellos }\end{array}$ \\
\hline 7 & $\begin{array}{c}\text { ANALISIS } \\
\text { ENCUESTAS Y } \\
\text { ENTREVISTAS }\end{array}$ & & $\begin{array}{l}\text { Análisis de resultados de las encuestas y } \\
\text { entrevistas estableciendo relaciones causales a } \\
\text { las diferentes problemáticas identificadas }\end{array}$ \\
\hline
\end{tabular}


Bio - grafía. Escritos sobre la Biología y su Enseñanza. ISSN 2027-1034

Edición Extraordinaria. p.p. 237 - 244

Memorias del IX Encuentro Nacional de Experiencias en Enseñanza de la Biología y la Educación Ambiental. IV Congreso Nacional de Investigación en Enseñanza de la Biología.

\begin{tabular}{|c|c|c|c|}
\hline \multicolumn{4}{|c|}{ Momento 2 : Diálogo de saberes } \\
\hline $\begin{array}{l}\text { Ses } \\
\text { ión }\end{array}$ & Nombre & Objetivo & Actividad \\
\hline 1 & $\begin{array}{l}\text { LLUVIA DE } \\
\quad \text { IDEAS }\end{array}$ & \multirow{3}{*}{$\begin{array}{lrr}\text { Generar } & & \text { posturas } \\
\text { críticas y propositivas } \\
\text { frente } \\
\text { problemáticas a las } \\
\text { ambientales detectadas }\end{array}$} & $\begin{array}{l}\text { Plenaria de lluvia de ideas sobre las } \\
\text { situaciones ambientales detectadas. }\end{array}$ \\
\hline 2 & $\begin{array}{l}\text { ÁRBOLDE } \\
\text { PROBLEMAS Y } \\
\text { SOLUCIONES }\end{array}$ & & $\begin{array}{l}\text { Construcción de árbol de problemas y de } \\
\text { potencialidades ambientales }\end{array}$ \\
\hline 3 & BIODANZA & & Biodanza de reconocimiento del entorno \\
\hline 4 & $\begin{array}{l}\text { VIDEO RUTA } \\
\text { DEL AGUA }\end{array}$ & \multirow{3}{*}{$\begin{array}{l}\text { Desarrollar talleres y } \\
\text { capacitaciones a los } \\
\text { integrantes del Club } \\
\text { Ambiental sobre el uso } \\
\text { racional y sostenible de } \\
\text { los recursos naturales. }\end{array}$} & $\begin{array}{l}\text { Video taller sobre problemas ambientales } \\
\text { contemporáneos. }\end{array}$ \\
\hline 5 & CINEFORO & & $\begin{array}{l}\text { Cine foro película el día después de mañana } \\
\text { como invitación a la reflexión sobre el } \\
\text { calentamiento global }\end{array}$ \\
\hline 6 & $\begin{array}{l}\text { TALLER LIBRO } \\
\text { ECO S.O.S. }\end{array}$ & & $\begin{array}{l}\text { Capacitación sobre manejo de residuos } \\
\text { sólidos con personal especializado }\end{array}$ \\
\hline 7 & $\begin{array}{c}\text { SALIDA } \\
\text { AMBIENTAL }\end{array}$ & \multirow{3}{*}{$\begin{array}{l}\text { Realizar diferentes } \\
\text { salidas pedagógicas a } \\
\text { escenarios ambientales } \\
\text { para reconocimiento de } \\
\text { formas de manejo } \\
\text { ambiental sostenible }\end{array}$} & $\begin{array}{l}\text { Salida a parque o escenario natural entre } \\
\text { nubes como taller de sensibilización } \\
\text { ambiental. }\end{array}$ \\
\hline 8 & $\begin{array}{l}\text { VISITA } \\
\text { COLEGIOS } \\
\text { CERCANOS }\end{array}$ & & $\begin{array}{l}\text { Visita a instituciones educativas, en las cuales } \\
\text { se realicen acciones de manejo ambiental } \\
\text { sostenible. }\end{array}$ \\
\hline 9 & $\begin{array}{l}\text { SALIDA JARDIN } \\
\text { BOTÁNICO }\end{array}$ & & $\begin{array}{l}\text { Salida pedagógica y taller agricultura urbana } \\
\text { en el Jardín Botánico de Bogotá }\end{array}$ \\
\hline
\end{tabular}

Para los dos otros momentos específicos se desarrolla esta misma planeación

\section{RESULTADOS Y DISCUSIÓN}

resultados destacados en términos de convenios y apoyos interinstitucionales así como participación en eventos y reconocimientos a nivel local, distrital y nacional, entre los que figuran: 
Bio - grafía. Escritos sobre la Biología y su Enseñanza. ISSN 2027-1034

Edición Extraordinaria. p.p. 237 - 244

Memorias del IX Encuentro Nacional de Experiencias en Enseñanza de la Biología y la

Educación Ambiental. IV Congreso Nacional de Investigación en Enseñanza de la Biología.

Convenios y apoyo interinstitucional

\begin{tabular}{|c|c|c|c|}
\hline ENTIDAD & PROGRAMA & ACTIVID AD & FECHA \\
\hline Colciencias & $\begin{array}{l}\text { Programa ONDAS: La } \\
\text { investigación como } \\
\text { estrategia pedagógica. }\end{array}$ & $\begin{array}{l}\text { Proyecto de investigación } \\
\text { ambiental "Calidad del aire } \\
\text { que respiramos en el colegio } \\
\text { Cundinamarca I.E.D." FASE } 1\end{array}$ & 2011-2012 \\
\hline Colciencias- UPN & $\begin{array}{l}\text { Programa ONDAS: La } \\
\text { investigación como } \\
\text { estrategia pedagógica. }\end{array}$ & $\begin{array}{l}\text { Proyecto de investigación } \\
\text { "¿De qué forma la agricultura } \\
\text { urbana contribuye a mejorar la } \\
\text { calidad del aire que } \\
\text { respiramos en el colegio } \\
\text { Cundinamarca?" FASE } 2\end{array}$ & 2013 \\
\hline $\begin{array}{l}\text { Hospital } \quad \text { Vista } \\
\text { Hermosa }\end{array}$ & $\begin{array}{l}\text { Proyecto de activación y } \\
\text { recuperación de zonas y } \\
\text { puntos críticos en la IED } \\
\text { Colegio Cundinamarca }\end{array}$ & $\begin{array}{l}\text { Mapas de zonas protectoras } \\
\text { Plantas físicas } 1 \text { a } 2 \text {. }\end{array}$ & 2013 \\
\hline Aseo Capital & $\begin{array}{l}\text { Proyecto de } \\
\text { Responsabilidad social y } \\
\text { ambiental }\end{array}$ & $\begin{array}{l}\text { Talleres sobre reciclaje y } \\
\text { reutilización. }\end{array}$ & 2016 \\
\hline $\begin{array}{l}\text { Alcaldía local de } \\
\text { Ciudad Bolívar y el } \\
\text { consorcio } \\
\text { Planear Proyectos }\end{array}$ & $\begin{array}{l}\text { Programa Basura cero en } \\
\text { la localidad de Ciudad } \\
\text { Bolívar }\end{array}$ & 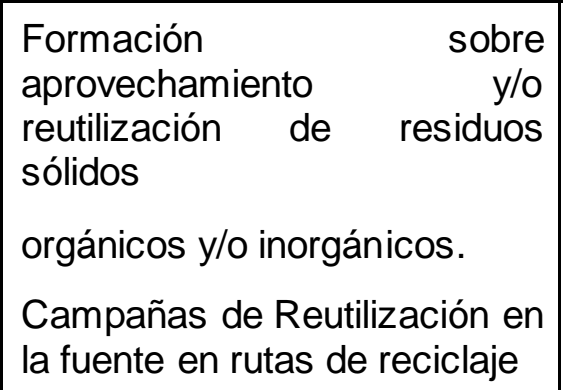 & 2016 \\
\hline Colciencias- UPN & $\begin{array}{l}\text { Programa ONDAS: La } \\
\text { investigación como } \\
\text { estrategia pedagógica. }\end{array}$ & $\begin{array}{l}\text { Proyecto de investigación } \\
\text { ambiental "Elaboración de } \\
\text { mapa de riesgos ambientales } \\
\text { externos del r colegio } \\
\text { Cundinamarca I.E.D.." FASE 3 }\end{array}$ & 2017 \\
\hline
\end{tabular}


Bio - grafía. Escritos sobre la Biología y su Enseñanza. ISSN 2027-1034

Edición Extraordinaria. p.p. 237 - 244

Memorias del IX Encuentro Nacional de Experiencias en Enseñanza de la Biología y la

Educación Ambiental. IV Congreso Nacional de Investigación en Enseñanza de la Biología.

\begin{tabular}{|l|l|l|l|l|}
\hline $\begin{array}{l}\text { Universidad Antonio } \\
\text { Nariño- Fac. } \\
\text { Ingeniería Ambiental }\end{array}$ & $\begin{array}{l}\text { Proyecto de grado } \\
\text { "Incidencia de la calidad } \\
\text { del aire en la salud de la } \\
\text { comunidad del Colegio } \\
\text { Cundinamarca }\end{array}$ & $\begin{array}{l}\text { Mediciones de manera in situ, } \\
\text { automático que se toman en } \\
\text { tiempo real, con equipos } \\
\text { portátiles }\end{array}$ & $2016-2017$ \\
\hline $\begin{array}{l}\text { Colegio } \\
\text { Cundinamarca I.E.D. } \\
\text { Grado 10 }\end{array}$ & $\begin{array}{l}\text { Anteproyectos de grado } \\
\text { con procesos de } \\
\text { investigación }\end{array}$ & $\begin{array}{l}\text { Línea de investigación. } \\
\text { "Calidad del aire" }\end{array}$ & 2017 \\
\hline
\end{tabular}

Además de estos resultados, se destacan las intervenciones favorables realizadas, las cuales han posibilitado el reconocimiento, intervención y transformación del entorno ambiental del colegio Cundinamarca, tales como

- Siembra y adopción de 50 árboles dentro y en los alrededores del colegio con apoyo del Jardín Botánico de Bogotá (2009)

- Diseño y construcción de unidad de agricultura urbana (2010)

- Ubicación de cercas vivas en algunas zonas verdes del colegio (2011)

- Talleres y prácticas de Fito medicina con apoyo del Hospital Vista Hermosa (2012)

- Prácticas de bioabono (Humus y compostaje) así como siembra de plantas aromáticas y hortalizas (2010-1017)

- Embellecimiento institucional con adecuación de jardineras (2013)

De igual forma en términos de avances curriculares y formativos para potenciar comunidades de saber pedagógico que posibiliten transformaciones al interior de la institución se destacan:

$\rightarrow$ Generación e implementación de la cátedra ambiente y desarrollo (2009-2012)

$\rightarrow$ Transversalizacion de la Educación Ambiental mediante las actividades del PRAE y del Club Ambiental.

$\rightarrow$ Fortalecimiento de valores en los estudiantes tales como el respeto, la autonomía, la solidaridad, la responsabilidad y el trabajo en equipo, a través de acciones como el cuestionamiento e intervención en su entorno.

$\rightarrow$ Desarrollo y potenciación del sentido de pertenencia institucional y ambiental al hacerse partícipes activos en la búsqueda de soluciones a problemáticas ambientales del colegio y su entorno.

Durante el tiempo en el cual se ha venido implementando la propuesta mediante este ambiente de aprendizaje...se fomenta el interés por aprender, asegurando el trabajo en equipo, garantizando la relación del contexto con el conocimiento" (SED 2015). Es así como la política pública en educación brinda unos lineamientos que, al ser abordados en clave de las necesidades de la comunidad educativa, generan mayor impacto que la misma formación disciplinar en horario regular. La jornada extendida, leída en clave de 
Bio - grafía. Escritos sobre la Biología y su Enseñanza. ISSN 2027-1034

Edición Extraordinaria. p.p. 237 - 244

Memorias del IX Encuentro Nacional de Experiencias en Enseñanza de la Biología y la

Educación Ambiental. IV Congreso Nacional de Investigación en Enseñanza de la Biología.

innovación, es un escenario potente en donde se optimizan los espacios, los recursos y las practicas pedagógicas.

\section{CONCLUSIONES}

Gracias a la implementación de este ambiente de aprendizaje denominado Club ambiental se ha logrado en cada una de las fases o momentos desarrollados:

- Fomentar y desarrollar en los estudiantes el desarrollo del pensamiento crítico al reconocer y reflexionar sobre su entorno ambiental, identificando fortalezas y debilidades.

- Fortalecer habilidades y actitudes de compromiso ambiental para el diseño e intervención a los problemas ambientales detectados.

- Consolidar un grupo de jóvenes líderes ambientales capaces de realizar actividades de impacto en la comunidad educativa tendientes a fomentar la sensibilización ambiental.

- Socializar los resultados obtenidos en los procesos de indagación e investigación ambiental mediante diferentes estrategias tales como direcciones de curso, pendones informativos, separadores de libros, pagina institucional y participación en feria Ondas (septiembre 2017).

\section{BIBLIOGRAFÍA}

COLEGIO CUNDINAMARCA I.E.D. Proyecto Ambiental Escolar PRAE. Valoración y manejo sostenible de los recursos de la institución. 2008.

JORNADA ESCOLAR 40 HORAS SEMANALES. (2014). Retrieved 2 August 2017, from http://idrd.gov.co/sitio/idrd/sites/default/files/imagenes/Jornada escolar 40 horas semanales.pdf

Ramírez María Soledad, Modelos y Estrategias para ambientes Innovadores, Editorial Digital, Monterrey México. 2012

SECRETARIA DE EDUCACIÓN DEL DISTRITO. Reorganización curricular por ciclos. Vol. 1. Ambientes de aprendizaje. 2015

SECRETARIA DE EDUCACIÓN DEL DISTRITO. Reorganización curricular por ciclos. Vol. 3. Ambientes de aprendizaje para el desarrollo humano. 2015

SECRETARIA DE EDUCACIÓN DEL DISTRITO. Currículo para la excelencia académica y la formación integral, Orientaciones generales. 2014

Verenzuela, Julia Rattia. ¿Pedagogía Crítica-Pedagogía Radical? Comunidad y Salud, vol. 6, núm. 1, Universidad de Carabobo Maracay, Venezuela. 2008. 